\title{
Acetylome analysis of the feline small intestine following Toxoplasma gondii infection
}

\author{
Yu-Meng Meng ${ }^{1,2} \cdot$ Bin-Tao Zhai $^{2} \cdot$ Hany M. Elsheikha ${ }^{3} \cdot$ Shi-Chen Xie ${ }^{2} \cdot$ Ze-Xiang Wang $^{4} \cdot$ Quan Zhao $^{1}$. \\ Xing-Quan Zhu ${ }^{1,2,5}$ (D) Jun-Jun $\mathrm{He}^{2}$
}

Received: 28 May 2020 / Accepted: 7 September 2020 / Published online: 20 September 2020

(C) Springer-Verlag GmbH Germany, part of Springer Nature 2020

\begin{abstract}
Toxoplasma gondii is a protozoan parasite capable of infecting a large number of warm-blooded animals and causes serious health complications in immunocompromised patients. T. gondii infection of the feline small intestine is critical for the completion of the life cycle and transmission of $T$. gondii. Protein acetylation is an important posttranslational modification, which plays roles in the regulation of various cellular processes. Therefore, understanding of how $T$. gondii reprograms the protein acetylation status of feline definitive host can help to thwart the production and spread of $T$. gondii. Here, we used affinity enrichment and high-resolution liquid chromatography with tandem mass spectrometry to profile the alterations of the acetylome in cat small intestine 10 days after infection by $T$. gondii Prugniuad (Pru) strain. Our analysis showed that $T$. gondii induced significant changes in the acetylation of proteins in the cat intestine. We identified 2606 unique lysine acetylation sites in 1357 acetylated proteins. The levels of 334 acetylated peptides were downregulated, while the levels of 82 acetylated peptides were increased in the infected small intestine. The proteins with differentially acetylated peptides were particularly enriched in the bioenergeticsrelated processes, such as tricarboxylic acid cycle, oxidative phosphorylation, and oxidation-reduction. These results provide the first baseline of the global acetylome of feline small intestine following $T$. gondii infection and should facilitate further analysis of the role of acetylated protein in the pathogenesis of $T$. gondii infection in its definitive host.
\end{abstract}

Keywords Toxoplasma gondii $\cdot$ Posttranslational modification $\cdot$ Cat $\cdot$ Lysine acetylation

\section{Introduction}

Toxoplasma gondii is an obligate intracellular pathogen that has been estimated to chronically infect approximately one-

Handling Editor: Julia Walochnik

Electronic supplementary material The online version of this article (https://doi.org/10.1007/s00436-020-06880-4) contains supplementary material, which is available to authorized users.

Xing-Quan Zhu

xingquanzhu1@hotmail.com

Jun-Jun He

hejunjun@caas.cn

1 College of Animal Science and Technology, Jilin Agricultural University, Changchun, Jilin Province 130118, People's Republic of China

2 State Key Laboratory of Veterinary Etiological Biology, Key Laboratory of Veterinary Parasitology of Gansu Province, Lanzhou Veterinary Research Institute, Chinese Academy of Agricultural Sciences, Lanzhou, Gansu 730046, People's Republic of China third of the world's population (Dubey 2010). However, $T$. gondii causes severe diseases in prenatally infected children and in immunocompromised individuals (Hohlfeld et al. 1989; Luft and Remington 1992). Although T. gondii has a
3 Faculty of Medicine and Health Sciences, School of Veterinary Medicine and Science, University of Nottingham, Sutton Bonington Campus, Loughborough LE12 5RD, UK

4 College of Veterinary Medicine, Gansu Agricultural University, Lanzhou, Gansu Province 730070, People's Republic of China

5 College of Veterinary Medicine, Shanxi Agricultural University, Taigu, Shanxi Province 030801, People's Republic of China 
wide range of intermediate hosts, felids are the only definitive host of this parasite. Cats play an important role in spreading of $T$. gondii, and one infected cat can discharge millions of oocysts in the feces (/Dubey 2001). The ability of $T$. gondii to enter and sexually differentiate within intestinal epithelial cells into oocysts constitutes a fundamental step in the biology and transmission of this parasite. A deeper understanding of the molecular events that shape the interaction of $T$. gondii with the feline intestine is therefore important for developing new intervention to interrupt the production of oocysts, which in turn may reduce the spread of infection to other hosts.

Protein acetylation was firstly discovered in 1964 (Allfrey et al. 1964) and participated in multiple cellular processes such as gene regulation, protein degradation, cellular metabolism, and cellular stress response (Lee and Gu 2013; Lima et al. 2011; Sang et al. 2017). Protein lysine acetylation (LysAc) has been reported in bacteria [e.g. Escherichia coli (Zhang et al. 2009), Bacillus subtilis (Kim et al. 2013)], parasites [e.g. Plasmodium falciparum (Miao et al. 2013)], insect [e.g. Drosophila melanogaster (Weinert et al. 2011)], and mammals [e.g. Mus musculus (Lundby et al. 2012) and Homo sapiens (Choudhary et al. 2009)]. These studies showed that acetylated proteins can influence a broad variety of cellular processes.

Acetylation of protein was found to play a role in regulation of the interaction between host and pathogen (Husain and Cheung 2014). A genome-wide acetylation analysis identified many acetylated proteins involved in diverse cellular processes and different localizations in $T$. gondii, suggesting potential of the parasite to modulate host cell acetylome (Jeffers and Sullivan, 2012). Lysine acetylation has also been studied in different genotype of $T$. gondii and was found to correlate with the virulence of the parasite strains (Wang et al. 2019). However, to what extent does $T$. gondii influence the cat intestine acetylome remains unknown.

In the present study, a quantitative analysis of the acetylome of cat small intestine infected by $T$. gondii was performed. To our knowledge, this is the first study to profile the acetylome alteration of feline small intestine following T. gondii infection.

\section{Materials and methods}

\section{Animals}

Six domestic cats (7- to 9-month-old) of the Chinese Li Hua breed were purchased from a local breeder and housed in a controlled environment. Prior to the experiment, sera of all cats were tested using a modified agglutination test (MAT) to confirm that the cats were negative for $T$. gondii antibodies. Commercial ELISA Kits (NuoYuan, Shanghai, China) were used to confirm that the cats used in the study were free of four viral infections (feline immunodeficiency virus, feline parvovirus, feline coronavirus, and feline leukemia virus). All cats had access to commercial diet and water ad libitum.

\section{Parasite infection and sample collection}

PRU strain (genotype II) of $T$. gondii was used in the present study. The parasite tissue cysts were collected from the brains of Kunming mice infected by PRU strain and counted microscopically. Six cats were randomly allocated to 2 groups (3 cats per group). Each cat in the infected group was orally inoculated with 100 cysts suspended in $1 \mathrm{ml}$ of sterile phosphate buffer saline (PBS), whereas cats of the control (noninfected) group received $1 \mathrm{ml}$ of sterile PBS only. Ten days postinfection, cats were euthanized, and their small intestines were collected followed by several washes using PBS to remove the intestinal contents. The collected intestinal samples were frozen immediately in liquid nitrogen and stored at $80{ }^{\circ} \mathrm{C}$ until use.

\section{Confirmation of $T$. gondii infection in the intestines}

Genomic DNA of harvested small intestine was extracted using TIANamp Genomic DNA Kit (TianGen ${ }^{\mathrm{TM}}$, Beijing, China). A nested PCR was used to detect $T$. gondii infection in cat intestine tissues as described previously (Lin et al. 2000). In the first round of the nested PCR, the following primers were used: TOXO1: 5'-GGAACTGCATCCGT TCATGAG-3' and TOXO2: 5'-TCTTTAAAGCGTTC GTGGTC-3'. PCR conditions included an initial denaturation step at $94{ }^{\circ} \mathrm{C}$ for $10 \mathrm{~min}$, followed by 30 PCR cycles of $94{ }^{\circ} \mathrm{C}$ for $1 \mathrm{~min}, 60^{\circ} \mathrm{C}$ for $15 \mathrm{~s}$, and $72{ }^{\circ} \mathrm{C}$ for $45 \mathrm{~s}$. In the second round of nested PCR, $1 \mu$ l PCR product $(\sim 193 \mathrm{bp})$ of first round was used as PCR template and the same protocol used in first round was followed, but with using primer TOXO 3 (5'-TGCATAGGTTGCAGTCACTG-3') instead of the primer TOXO 1 with anticipated amplicon size of $131 \mathrm{bp}$. The PCR products were electrophoresed on $1 \%$ agarose-Trisacetate-EDTA gel that was stained with ethidium bromide. The PCR products were purified and sequenced. Also, fecal samples were collected daily and examined using flotation method to monitor the shedding of $T$. gondii oocysts.

\section{Protein extraction}

The appropriate amount of lysis buffer (containing $8 \mathrm{M}$ urea and protease inhibitor) was added to each sample followed by sonication on ice for $5 \mathrm{~min}$. Then, samples were centrifuged at $25,000 \mathrm{~g}$ for $20 \mathrm{~min}$ at $4{ }^{\circ} \mathrm{C}$, and the supernatant was collected. Dithiothreitol (DTT) was added to the supernatant at a final concentration of $10 \mathrm{mM}$, and the samples were incubated at 56 ${ }^{\circ} \mathrm{C}$ for $1 \mathrm{~h}$ in a water bath. The deacetylase inhibitor $(55 \mathrm{mM}$ IAM) was added followed by incubation in the dark for 
$45 \mathrm{~min}$ at room temperature. Finally, protein concentration of each protein sample was determined by using the Bradford assay.

\section{Trypsin digestion and affinity enrichment of acetylated peptides}

From each sample, $5 \mathrm{mg}$ of extracted protein were diluted with $30 \mathrm{mM}$ HEPES until the final concentration of urea reached < $2 \mathrm{M}$. Then, trypsin was added at protein to trypsin mass ratio of $50: 1$ and digested at $37^{\circ} \mathrm{C}$ for overnight. The digested peptides were desalted using Sep-Pac C18 column and vacuum dried. The tryptic peptides were dissolved in IAP buffer and mixed with the coupled acetylated antibody beads at $4{ }^{\circ} \mathrm{C}$ overnight with gentle shaking. The sample was centrifuged at $2000 \mathrm{~g}$ and $4{ }^{\circ} \mathrm{C}$ for $4 \mathrm{~s}$, and the supernatant was discarded. The sediment was washed twice with $1 \mathrm{ml}$ IPA buffer followed by washing three times with precooled double distilled $\mathrm{H}_{2} \mathrm{O}$. After adding $200 \mu 10.15 \%$ trifluoroacetic acid (TFA) and incubation for $10 \mathrm{~min}$ at room temperature, the sample was centrifuged at $2000 \mathrm{~g}$ and $4{ }^{\circ} \mathrm{C}$ for $4 \mathrm{~s}$, and the supernatant was collected. Finally, the peptides in the supernatant were dried using a vacuum centrifuge.

\section{HPLC fractionation and LC-MS/MS analysis}

The dried peptides were reconstituted in double distilled $\mathrm{H}_{2} \mathrm{O}$ with $2 \% \mathrm{ACN}$ and $0.1 \% \mathrm{FA}$. Then, the dissolved peptides were separated by UHPLC (UltiMate 3000, Thermo) using the HPLC gradient elution settings described in Table S1. Peptide segments were ionized by nanoESI source and then detected by tandem mass spectrometer Q-Exactive HF (Thermo Fisher Scientific, San Jose, CA) with DDA (datadependent acquisition) detection mode. The $\mathrm{m} / \mathrm{z}$ scan range was 350 to 1500 for full scan, and intact peptides were detected in the orbitrap at a resolution of 120,000. The analyses of the MS/MS data, including identification, normalization, and differential analysis, were processed using MaxQuant (v.1.5.3.30) search engine (Cox and Mann 2008). Tandem mass spectra were searched against UniProt database for protein identification. Trypsin/P was specified as cleavage enzyme allowing up to 4 missing cleavages. The mass tolerance for precursor ions was set as $20 \mathrm{ppm}$ and $0.05 \mathrm{Da}$ for fragment ions. Carbamidomethylation of cysteine was considered as a fixed modification. Methionine oxidation, acetylation on lysine, and acetylation on protein $\mathrm{N}$-terminal were designated as variable modifications. The false discovery rate threshold (FDR) was set as $1 \%$. All other parameters in MaxQuant were set to the default values. The site location probability was set as $>0.75$. The threshold of differentially acetylated peptides (DAPs) was $P<0.05$.

\section{Bioinformatics analysis}

Gene ontology (GO) analysis was performed to identify the enriched acetylated proteins among the proteins with differentially acetylated peptides (PDAPs) by using ToxoDB 11.0 and UniProt-GOA database, and acetylated proteins were classed into three categories: molecular function (MF), biological process (BP), and cellular component (CC). The orthologous proteins were classified by using orthologous groups of proteins (COG) database (Tatusov et al. 2000). Functional enrichment analysis was performed to identify the enriched pathways using Kyoto Encyclopedia of Genes and Genomes (KEGG) database (Kanehisa 2008). KEGG pathways were considered significant at a $P$ value of $<0.05$. WoLF PSORT algorithm was applied to predict the subcellular locations of the PDAPs (Horton et al. 2007). For motif analysis, the 11mers amino acid ( \pm 5 amino acids residues around the lysine acetylation site) of sequences of all DAPs was analyzed using Motif-x software. All protein sequences were used as background and other parameters were set to the default values.

\section{Results}

\section{Confirmation of $T$. gondii infection and overall features of the differentially acetylated peptides}

Daily fecal examination detected $T$. gondii oocysts in feline feces at 7 dpi. All feline small intestines were PCR positive (Figure S1), and our DNA sequencing confirmed that the PCR product was $\mathrm{B} 1$ gene of $T$. gondii. In high-resolution immunoaffinity LC-MS/MS analysis, the mass errors were less than $6 \mathrm{ppm}$, and the majority were close to zero (Fig. 1a), showing the accuracy of the data obtained in the present study. A total of 2606 unique acetylation sites of 1357 proteins were identified (Table S2). Acetylated peptides with $P$ value $<0.05$ were deemed as differentially regulated. In this study, the acetylation of 334 peptides was decreased in the infected small intestine, whereas acetylation of 82 peptides was increased (Fig. $1 \mathrm{~b}$ and Table S3). About $65 \%$ acetylated proteins had only one acetylated site, $17 \%$ acetylated proteins contained two acetylated site, $10 \%$ acetylated proteins contained three or four acetylated sites, and $8 \%$ acetylated proteins contained five or more acetylated sites (Fig. 1c). To identify the motif of DAPs in feline small intestine, the character of the amino acid sequence adjacent to acetylation sites was analyzed. As shown in Fig. 1d, no clear acetylation motif was detected.

\section{Functional classification and subcellular localization of the proteins with differentially acetylated peptides}

To elucidate the functions of PDAPs altered by $T$. gondii infection in the small intestine, subcellular localization, GO, 
a

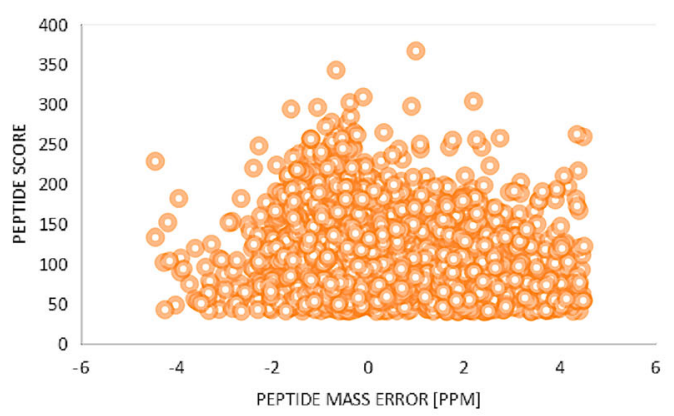

C

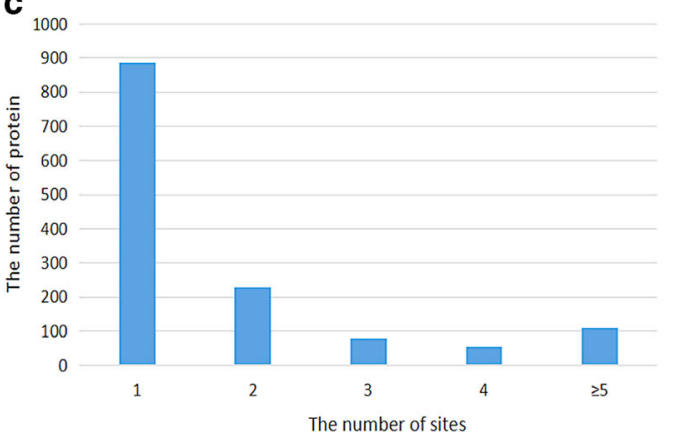

Fig. 1 The properties of the acetylated peptides of cat small intestine infected by Toxoplasma gondii. a Mass error distribution of all identified peptides. b Volcano plot of the differentially acetylated peptides (DAPs). DAPs are shown as a red (upregulated) or green d
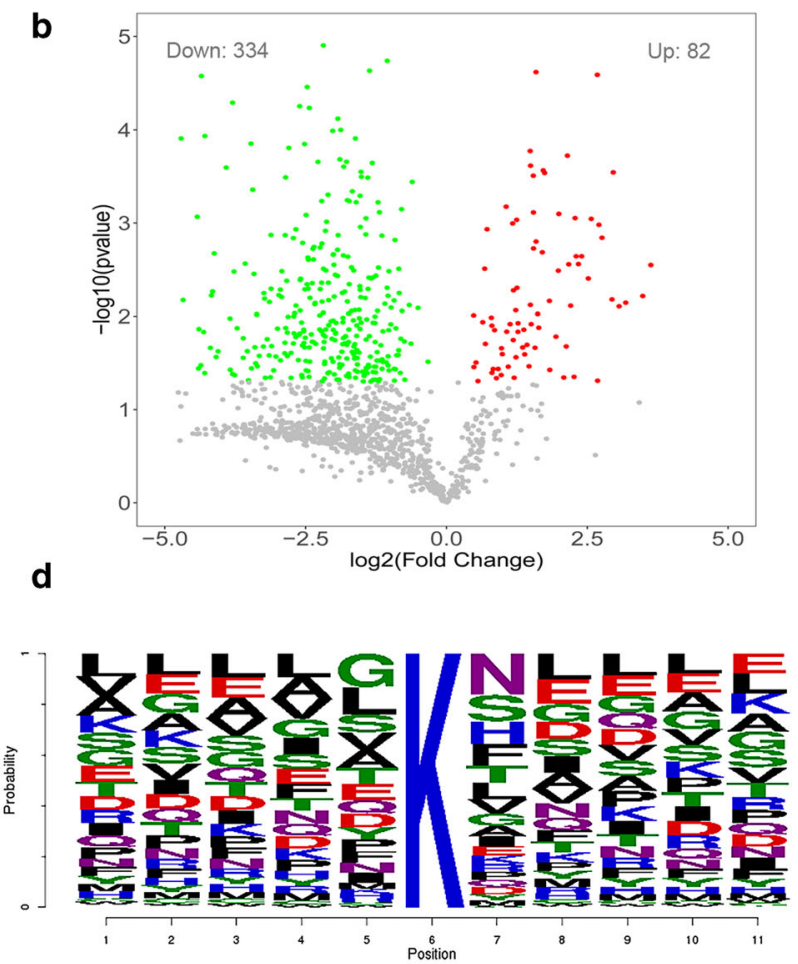

(downregulated) dot. The acetylated peptides that did not show any significant alteration between infected and control are shown as grey dots. $\mathbf{c}$ The number of acetylation sites per protein. $\mathbf{d}$ Sequence probability logo of acetylation motif for +10 amino acids adjacent to acetylation site
KEGG, and COG classification analyses were performed. Analysis of subcellular localization of PDAPs indicated that most PDAPs were localized in the cytoplasm (32\%), nucleus (29\%), mitochondria (18\%), extracellular (8\%), cyto-nucl (3\%), and endoplasmic reticulum (2\%) (Fig. 2a).

COG analysis showed that PDAPs were classified into 24 functional groups. The proteins involved in translation, ribosomal structure and biogenesis, posttranslational modification, protein turnover, and chaperones account for a large proportion of the PDAPs (Fig. 2b). According to COG annotations, a number of metabolically related enzymes were identified as acetylated proteins, including acyl-coenzyme A oxidase 3, aconitate hydratase, malate dehydrogenase, isocitrate dehydrogenase, dihydrolipoyl dehydrogenase, fumarate hydratase, and citrate synthase.

To further identify intestinal biological changes that may have been influenced by PDAPs induced by $T$. gondii, we performed GO and KEGG pathway enrichment analyses with all PDAPs. GO enrichment identified several PDAPs involved in mitochondrial matrix (6.92\%), mitochondrion $(6.11 \%)$, and organelle part $(3.52 \%)$. This result was consistent with that of WoLF PSORT prediction. Also, a variety of molecular functions were enriched, such as actin-dependent ATPase activity (3.22\%), cysteine-type endopeptidase activity (2.88\%), anion channel activity (2.66\%), and oxidoreductase activity (2.54\%), which were involved in oxidation-reduction process $(4.55 \%)$ and various metabolic process $(\geq 2.98 \%)$ (Fig. $3 \mathrm{a}$ and Table S4). According to the KEGG enrichment analysis, a majority of the PDAPs were related to microbial metabolism, carbon metabolism, fatty acid metabolism, and degradation (Fig. 3b).

For proteins with upregulated acetylated peptides (Fig. 4a), DNA binding, zinc ion binding, and protein binding were the top $3 \mathrm{GO}$ terms in the MF category. In the CC category, most proteins with upregulated acetylated peptides were enriched in nuclear and nucleolar. The positive regulation of nucleosome assembly and apoptosis in the BP category corresponds to a relatively large number of proteins with upregulated acetylated peptides. Regarding the proteins with downregulated acetylated peptides (Fig. 4b), the results of MF indicated that the largest group was ATP-binding protein, and the second largest group was protein-binding protein. For the $\mathrm{CC}$ category, most proteins with downregulated acetylated peptides correspond to cytoplasm and mitochondria. In the BP category, the redox process and the ATP decomposition process were the top two protein groups enriched by the proteins with downregulated acetylated peptides. 
Fig. 2 Functional classification of protein with differentially acetylated peptides (PDAPs) in cat small intestine infected by Toxoplasma gondii. a Pie chart showing the percentage and subcellular localization of the PDAPs. b Functional categories of PDAPs based on clusters of orthologous group (COG) annotation

\section{a}

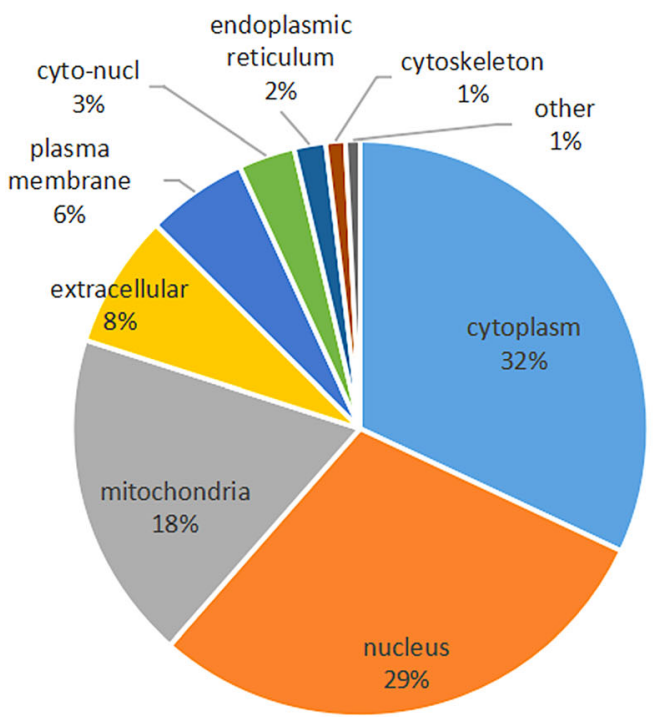

b Cog Function Classification of Scaffold Sequence

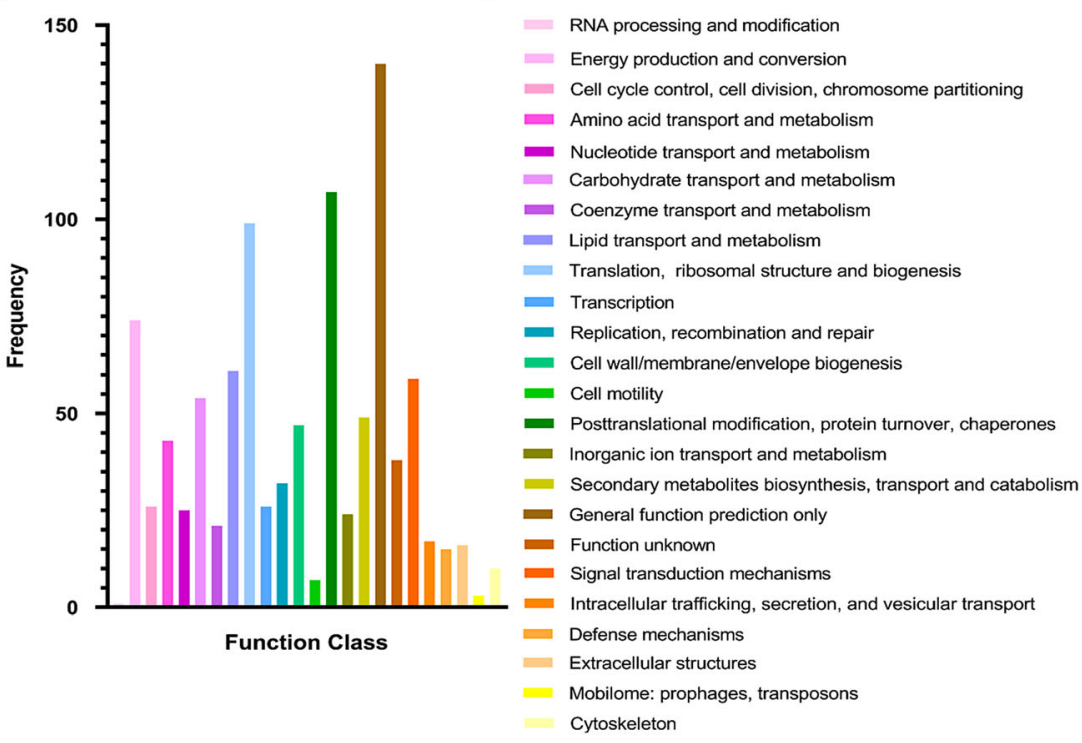

\section{Identification of acetylated proteins of $T$. gondii}

In the present study, we identified 40 acetylated proteins of T. gondii, including 64 acetylated peptides (Table S3). These proteins mainly included histones and heat shock proteins. In total, we found 21 novel acetylated proteins that have not been identified previously (Wang et al. 2019). These novel acetylated proteins included TGME49_286470, TGME49_206430, TGME49_214260, TGME49_313830, TGME49_306240, TGME49_213770, TGME49_300612, TGME49_207770, TGME49_305240, TGME49_245530, TGME49_268380, TGME49_211890, TGME49_241300, TGME49_207100, TGME49_254050, TGME49_286470, TGME49 231815, TGME49 217220, TGME49 253360, TGME49_282210, and TGME49_201640. Among these novel acetylated proteins, the acetylated hypothetical protein TGME49_201640 is only expressed in the oocyst and feline enterocyte stage. The function of TGME49_201640 remains unknown; however, the protein has one signal peptide at $\mathrm{N}$ terminal. WoLF PSORT prediction showed that the protein is a secretory protein.

\section{Discussion}

The aim of this study was to analyze the effect of $T$. gondii infection on lysine acetylation profile of small intestinal protein in cats. Compared with the control (uninfected) cats, a total of 2606 unique acetylated sites in 1357 proteins were detected in the feline small intestine attributed to the T. gondii infection. Most of these proteins have only a single lysine acetylation site. These results agree with that reported previously (Weinert et al. 2011; Zhou et al. 2016a). T. gondii infection can alter the expression of genes involved in protein 


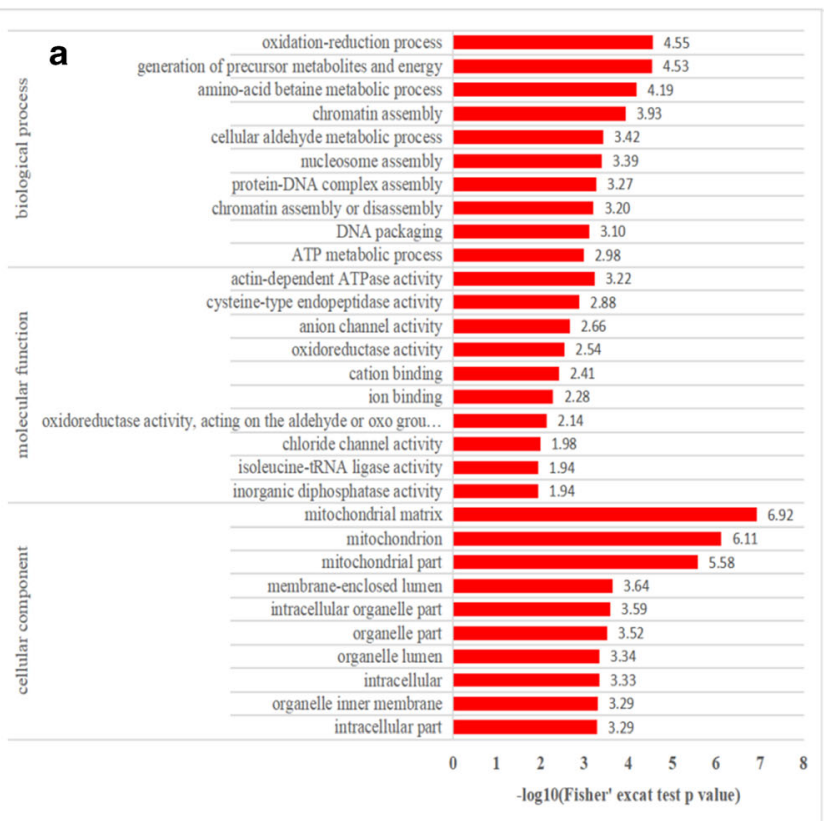

Fig. 3 GO enrichment analysis and KEGG pathway analysis of all PDAPs of cat small intestine infected by Toxoplasma gondii. a Top 30 enriched GO terms categorized according to Biological process, molecular function and cellular component. b Scatterplot of the top 20

modification processes in feline small intestine (Cong et al. 2018; Wang et al. 2018). Thus, it is possible that the lack of clear acetylation motif in this study could be due to alteration in the genes participating in ubiquitous acetylation process by T. gondii infection.

GO enrichment and KEGG pathway analyses showed that the proteins with differentially acetylated peptides (PDAPs) were significantly enriched in metabolic processes, such as redox processes and peroxisomes (Fig. 3). This is rather anticipated because infection by $T$. gondii is associated with considerable metabolic changes of the infected host (Zhou

\section{a}

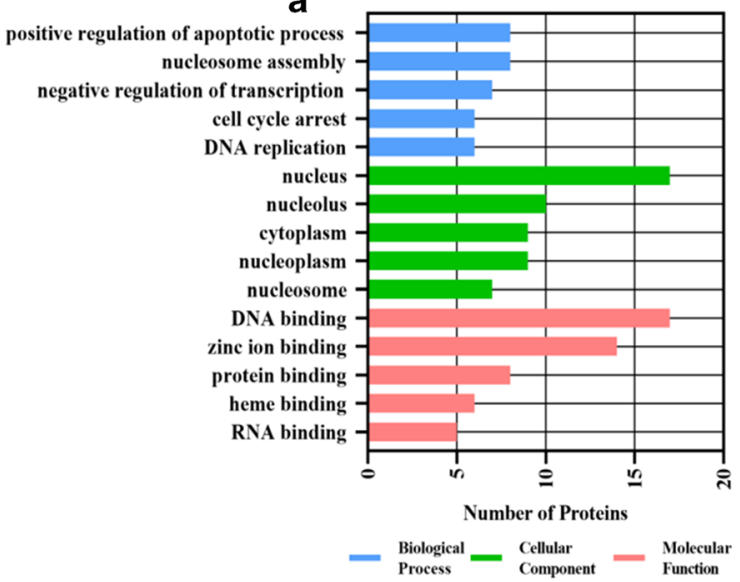

Fig. 4 Gene Ontology functional analysis of the proteins with increased or decreased acetylated peptide abundance categorized according to biological process, molecular function and cellular component. a GO

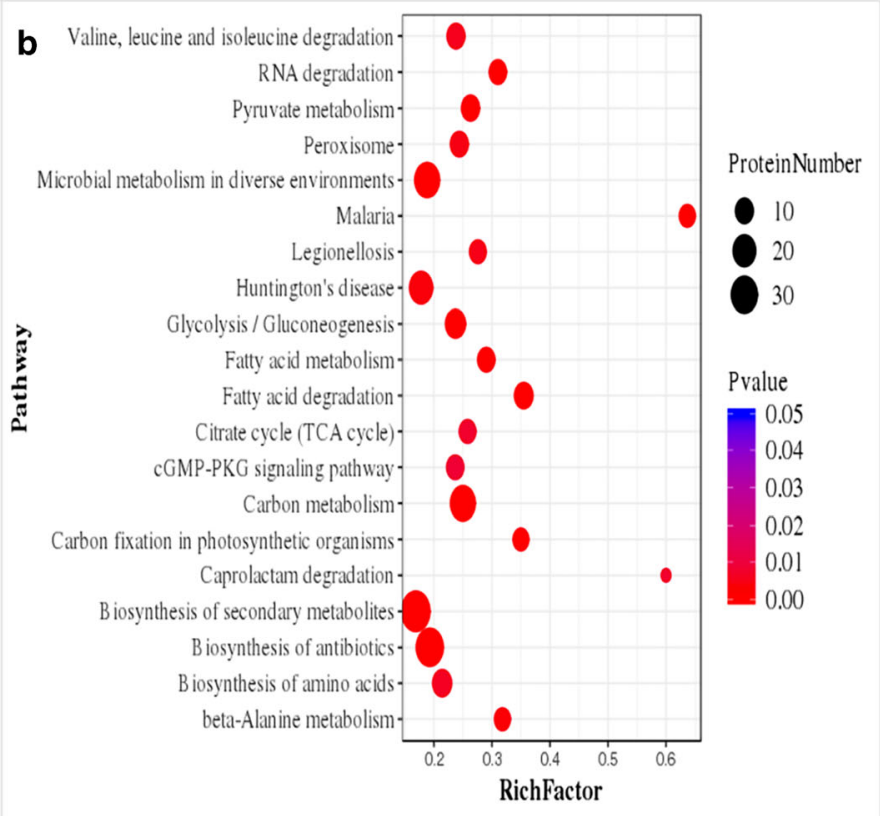

enriched KEGG pathways of the PDAPs. The rich factor on the $x$-axis represents the ratio of PDAP's number to the total number of proteins annotated in the pathway

et al. 2015, 2016b). Previous proteomic studies have shown that a large number of protein lysine acetylation events occur in the mitochondria (Gibson 2005; Jardim et al. 2018; Peinado et al. 2014). As shown in Fig. 4b, mitochondrion was enriched by the proteins with downregulated acetylated peptides. In TCA cycle, malate dehydrogenase (MDH) and aconitate hydratase $(\mathrm{ACO} 2)$ whose structural and functional stability had impacts on mitochondrial energy metabolism (Matasova and Popova 2008; Wang et al. 2009) were highly acetylated. In TCA cycle, citrate synthase (CS), isocitrate dehydrogenase (IDH), and oxoglutarate dehydrogenase complex (OGDC) are

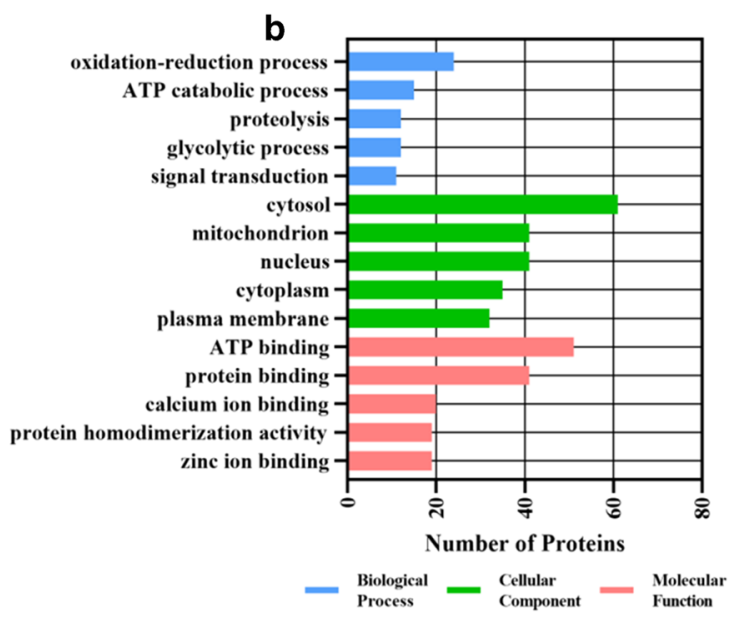

functional classification of the protein with increased acetylated peptide abundance. b GO functional classification of the protein with decreased acetylated peptide abundance 
rate-limiting enzymes that play roles in regulating the TCA process. TCA cycle and oxidative phosphorylation (OxPhos) are crucial steps for ATP production. In the present study, 44 proteins whose acetylated peptide abundances were downregulated in the infected small intestine were enriched in the OxPhos process (Fig. S2). The downregulated acetylation of OxPhos process and hypometabolic state seems to be bioenergetic tradeoffs strategy to promote host tissue tolerance to infection.

Peroxisome is an organelle involved in metabolic and stress responses (Cavallini et al. 2017; Mast et al. 2015). It functions as a hub for immune metabolism, supporting cellular metabolites to control the development and activation of immune cells and regulating inflammatory pathways in different immune cells (Di Cara et al. 2019). The KEGG pathway enrichment analysis showed that, in T. gondii-infected small intestine, 10 proteins with downregulated acetylated peptides were associated with peroxisomes (Fig. S3). These proteins included hydroxysteroid dehydrogenase, catalase, isocitrate dehydrogenase, carnitine O-acetyltransferase, acyl-coenzyme A oxidase, superoxide dismutase, peroxiredoxin, nudix hydrolase 13, ATP binding cassette subfamily D member 3, and acyl-CoA synthetase long chain family member 4 . Acyl coenzyme A oxidase has been shown to drive the peroxisome $\beta$ oxidation and to play an important role in inflammation control (Vamecq et al. 2018). These results indicate the involvement of peroxisome in antiT. gondii immune responses.

T. gondii has been shown to influence the host cell cycle (Elsheikha et al. 2019) and subvert apoptosis signaling in host cells to promote its own survival (Lüder and Gross 2005; He et al. 2016; Cong et al. 2017). GO annotation analysis showed that most of the proteins with increased acetylated peptides are involved in promoting apoptosis (Fig. 4a). Interestingly, GO enrichment uncovered several PDAPs involved in mitochondrial matrix and mitochondrion. Also, we have shown in this study that $T$. gondii through downregulation of the acetylation of OxPhos can affect mitochondrial metabolism. The cytochrome $c$ protein, which controls redox signaling in mitochondrial OxPhos, is released from the mitochondria into the cytosol to activate caspase proteases that trigger apoptotic changes (Hengartner 2000). These results suggest a clear impact of $T$. gondii-induced alterations of protein acetylation on the bioenergetics and apoptotic processes in the cat intestine. Sexual reproduction of $T$. gondii is a highly elaborate event, which involves parasite proliferation, differentiation and host cell destruction (Dubey et al. 1998). Following invasion of the feline small intestine, tachyzoites transform into a presexual merozoite that replicates and differentiates into male and female gametes, which ultimately form zygotes. Therefore, it is reasonable to anticipate a significant metabolic burden and an increased cellular destruction during $T$. gondii infection of the feline intestine. To what extent the acetylated peptides that were found associated with apoptosis and mitochondrial metabolism in the present study can influence the intrinsic and extrinsic apoptotic pathways in the cat small intestine remains to be elucidated.

The acetylated protein of $T$. gondii tachyzoites has been described previously (Wang et al. 2019). However, the acetylation status of $T$. gondii proteins during intestinal infection of cats was unknown until the present study, where we also identified 40 acetylated proteins of T. gondii. The majority of the acetylated proteins of T. gondii belong to histone $\mathrm{H} 2 \mathrm{~A}$, histone $\mathrm{H} 2 \mathrm{~B}$, histone $\mathrm{H} 3$, and histone $\mathrm{H} 4$ families. Histone is an important component of chromatin and plays a key role in regulation of gene expression (Nardelli et al. 2013). A previous report showed that shock protein 70 (HSP70) and heat shock protein 10 (HSP10) of $T$. gondii are acetylated proteins (Wang et al. 2019). In the present study, HSP70 and HSP10 were also identified as acetylated proteins. HSP70 is a virulence factor expressed during the transformation of $T$. gondii from tachyzoites to bradyzoites and plays a role in parasite replication and immune responses (Barenco et al. 2014). Also, we found one acetylated hypothetical protein TGME49_201640 whose function remains unknown and is only expressed in the oocyst and feline enterocyte stage. Bioinformatic analysis showed that this protein is a secretory protein. This suggests that the product of the TGME49_201640 gene might be discharged into host cells and contributes to the interaction between feline enterocyte and $T$. gondii. The identified acetylated peptides listed in Table S3 may help us to elucidate new biological functions of $T$. gondii secretome in mediating the interaction between $T$. gondii and the small intestine of the feline definitive host.

\section{Conclusion}

This study, for the first time, showed that $T$. gondii infection had significant effect on the acetylome of cat small intestine. High-resolution mass spectrometry-based proteomics approach identified 2606 acetylation sites in 1357 acetylated proteins. Acetylated proteins altered by infection were found to belong to different cell compartments (cytoplasm, nucleus, mitochondria, extracellular component) and to participate in various metabolic pathways or cellular processes (glycolysis/ gluconeogenesis, citrate cycle (TCA cycle) and peroxisome). We identified a list of novel acetylated proteins of $T$. gondii, including one hypothetical protein that is only expressed at the oocyst and feline enterocyte stage. Our data provided new and valuable resource to further investigations of the role of protein acetylation in the sexual reproduction of $T$. gondii in feline small intestine. 
Acknowledgments We thank Novogene Co. Ltd (Beijing) for technical assistance.

Availability of data and material The mass spectrometry data have been submitted to the iProX database with the project ID IPX0002163000.

Funding Project support was kindly provided by the National Natural Science Foundation of China (Grant No. 31902291), the International Science and Technology Cooperation Project of Gansu Provincial Key Research and Development Program (Grant No. 17JR7WA031), and the Agricultural Science and Technology Innovation Program (ASTIP) (Grant No. CAAS-ASTIP-2016-LVRI-03).

\section{Compliance with ethical standards}

All animals were handled strictly according to the Animal Ethics Procedures and Guidelines of the People's Republic of China. The study was reviewed and approved by the Animal Administration and Ethics Committee of Lanzhou Veterinary Study Institute, Chinese Academy of Agricultural Sciences.

Conflict of interest The authors declare that they have no competing interests.

\section{References}

Allfrey VG, Faulkner R, Mirsky AE (1964) Acetylation and methylation of histones and their possible role in the regulation of RNA synthesis. Proc Natl Acad Sci U S A 51:786-794

Barenco PV, Lourenço EV, Cunha-Júnior JP, Almeida KC, RoqueBarreira MC, Silva DA, Araújo EC, Coutinho LB, Oliveira MC, Mineo TW, Mineo JR, Silva NM (2014) Toxoplasma gondii $70 \mathrm{kDa}$ heat shock protein: systemic detection is associated with the death of the parasites by the immune response and its increased expression in the brain is associated with parasite replication. PLoS One 9:e96527

Cavallini G, Donati A, Taddei M, Bergamini E (2017) Peroxisomes proliferation and pharmacological stimulation of autophagy in rat liver: evidence to support that autophagy may remove the "older" peroxisomes. Mol Cell Biochem 431:97-102

Choudhary C, Kumar C, Gnad F, Nielsen ML, Rehman M, Walther TC, Olsen JV, Mann M (2009) Lysine acetylation targets protein complexes and co-regulates major cellular functions. Science 325:834 840

Cong W, Zhang X, He J, Li F, Elsheikha HM, Zhu X (2017) Global miRNA expression profiling of domestic cat livers following acute Toxoplasma gondii infection. Oncotarget 8:25599-25611

Cong W, Dottorini T, Khan F, Emes RD, Zhang FK, Zhou CX, He JJ, Zhang XX, Elsheikha HM, Zhu XQ (2018) Acute Toxoplasma gondii infection in cats induced tissue-specific transcriptional response dominated by immune signatures. Front Immunol 9:2403

Cox J, Mann M (2008) MaxQuant enables high peptide identification rates, individualized p.p.b.-range mass accuracies and proteomewide protein quantification. Nat Biotechnol 26:1367-1372

Di Cara F, Andreoletti P, Trompier D, Vejux A, Bülow MH, Sellin J, Lizard G, Cherkaoui-Malki M, Savary S (2019) Peroxisomes in immune response and inflammation. Int J Mol Sci 20:3877

Dubey JP (2001) Oocyst shedding by cats fed isolated bradyzoites and comparison of infectivity of bradyzoites of the VEG strain Toxoplasma gondii to cats and mice. J Parasitol 87:215-219

Dubey JP (2010) Toxoplasmosis of animals and humans CRC Press. Raton, Boca
Dubey JP, Lindsay DS, Speer CA (1998) Structures of Toxoplasma gondii tachyzoites, bradyzoites, and sporozoites and biology and development of tissue cysts. Clin Microbiol Rev 11:267-299

Elsheikha HM, Elsaied NA, Chan KLA, Brignell C, Harun MSR, Wehbe $\mathrm{K}$, Cinquee $\mathrm{G}$ (2019) Label-free characterization of biochemical changes within human cells under parasite attack using synchrotron based micro-FTIR. Anal Methods 11:2518-2530

Gibson BW (2005) The human mitochondrial proteome: oxidative stress, protein modifications and oxidative phosphorylation. Int J Biochem Cell Biol 37:927-934

He JJ, Ma J, Elsheikha HM, Song HQ, Zhou DH, Zhu XQ (2016) Proteomic profiling of mouse liver following acute Toxoplasma gondii infection. PLoS One 11:e0152022

Hengartner MO (2000) The biochemistry of apoptosis. Nature 407:770 776

Hohlfeld P, Daffos F, Thulliez P, Aufrant C, Couvreur J, MacAleese J, Descombey D, Forestier F (1989) Fetal toxoplasmosis: outcome of pregnancy and infant follow-up after in utero treatment. J Pediatr 115:765-769

Horton P, Park KJ, Obayashi T, Fujita N, Harada H, Adams-Collier CJ, Nakai K (2007) WoLF PSORT: protein localization predictor. Nucleic Acids Res 35:W585-W587

Husain M, Cheung CY (2014) Histone deacetylase 6 inhibits influenza A virus release by downregulating the trafficking of viral components to the plasma membrane via its substrate, acetylated microtubules. J Virol 88:11229-11239

Jardim A, Hardie DB, Boitz J, Borchers CH (2018) Proteomic profiling of Leishmania donovani promastigote subcellular organelles. J Proteome Res 17:1194-1215

Jeffers V, Sullivan WJ (2012) Lysine acetylation is widespread on proteins of diverse function and localization in the protozoan parasite Toxoplasma gondii. Eukaryot Cell 11:735-742

Kanehisa M (2008) Bioinformatics and metabolome analysis: overview. Tanpakushitsu Kakusan Koso 53:1697

Kim D, Yu BJ, Kim JA, Lee YJ, Choi SG, Kang S, Pan JG (2013) The acetylproteome of gram-positive model bacterium Bacillus subtilis. Proteomics 13:1726-1736

Lee JT, Gu W (2013) SIRT1: Regulator of p53 deacetylation. Genes Cancer 4:112-117

Lima BP, Antelmann H, Gronau K, Chi BK, Becher D, Brinsmade SR, Wolfe AJ (2011) Involvement of protein acetylation in glucoseinduced transcription of a stress-responsive promoter. Mol Microbiol 81:1190-1204

Lin MH, Chen TC, Kuo TT, Tseng CC, Tseng CP (2000) Real-time PCR for quantitative detection of Toxoplasma gondii. J Clin Microbiol 38:4121-4125

Lüder C, Gross U (2005) Apoptosis and its modulation during infection with Toxoplasma gondii: molecular mechanisms and role in pathogenesis. Curr Top Microbiol Immunol 289:219-237

Luft BJ, Remington JS (1992) Toxoplasmic encephalitis in AIDS. Clin Infect Dis 15:211-222

Lundby A, Lage K, Weinert BT, Bekker-Jensen DB, Secher A, Skovgaard T, Kelstrup CD, Dmytriyev A, Choudhary C, Lundby C, Olsen JV (2012) Proteomic analysis of lysine acetylation sites in rat tissues reveals organ specificity and subcellular patterns. Cell Rep 2:419-431

Mast FD, Rachubinski RA, Aitchison JD (2015) Signaling dynamics and peroxisomes. Curr Opin Cell Biol 35:131-136

Matasova LV, Popova TN (2008) Aconitate hydratase of mammals under oxidative stress. Biochemistry (Mosc) 73:957-964

Miao J, Lawrence M, Jeffers V, Zhao F, Parker D, Ge Y, Sullivan WJ, Cui L (2013) Extensive lysine acetylation occurs in evolutionarily conserved metabolic pathways and parasite-specific functions during Plasmodium falciparum intraerythrocytic development. Mol Microbiol 89:660-675 
Nardelli SC, Che FY, Silmon de Monerri NC, Xiao H, Nieves E, MadridAliste C, Angel SO, Sullivan WJ, Angeletti RH, Kim K, Weiss LM (2013) The histone code of Toxoplasma gondii comprises conserved and unique posttranslational modifications. mBio 4:e0922-e00913

Peinado JR, Diaz-Ruiz A, Fruhbeck G, Malagon MM (2014) Mitochondria in metabolic disease: getting clues from proteomic studies. Proteomics 14:452-466

Sang Y, Ren J, Qin R, Liu S, Cui Z, Cheng S, Liu X, Lu J, Tao J, Yao YF (2017) Acetylation regulating protein stability and DNA-binding ability of HilD, thus modulating Salmonella Typhimurium virulence. J Infect Dis 216:1018-1026

Tatusov RL, Galperin MY, Natale DA, Koonin EV (2000) The COG database: a tool for genome-scale analysis of protein functions and evolution. Nucleic Acids Res 28:33-36

Vamecq J, Andreoletti P, ElKebbaj R, Saih FE, Latruffe N, ElKebbaj MH, Lizard G, Nasser B, Cherkaoui-Malki M (2018) Peroxisomal acyl-coA oxidase type 1: anti-inflammatory and anti-aging properties with a special emphasis on studies with LPS and argan oil as a model transposable to aging. Oxidative Med Cell Longev 2018: 6986984

Wang XY, Wang B, Hou ST, Zhu GP (2009) Structure and function of malate dehydrogenases. J Biol 26:69-72

Wang M, Zhang FK, Elsheikha HM, Zhang NZ, He JJ, Luo JX, Zhu XQ (2018) Transcriptomic insights into the early host-pathogen interaction of cat intestine with Toxoplasma gondii. Parasit Vectors 11:592
Wang ZX, Hu RS, Zhou CX, He JJ, H ME, Zhu XQ (2019) Label-free quantitative acetylome analysisreveals Toxoplasma gondii genotype-specific acetylomic signatures. Microorganisms 7:510

Weinert BT, Wagner SA, Horn H, Henriksen P, Liu WR, Olsen JV, Jensen LJ, Choudhary C (2011) Proteome-wide mapping of the Drosophila acetylome demonstrates a high degree of conservation of lysine acetylation. Sci Signal 4:ra48

Zhang J, Sprung R, Pei J, Tan X, Kim S, Zhu H, Liu CF, Grishin NV, Zhao Y (2009) Lysine acetylation is a highly abundant and evolutionarily conserved modification in Escherichia coli. Mol Cell Proteomics 8:215-225

Zhou CX, Zhou DH, Elsheikha HM, Liu GX, Suo X, Zhu XQ (2015) Global metabolomic profiling of mice brains following experimental infection with the cyst-forming Toxoplasma gondii. PLoS One 10:e0139635

Zhou S, Yang Q, Yin C, Liu L, Liang W (2016a) Systematic analysis of the lysine acetylome in Fusarium graminearum. BMC Genomics 17: 1019

Zhou CX, Zhou DH, Elsheikha HM, Zhao Y, Suo X, Zhu XQ (2016b) Metabolomic profiling of mice serum during toxoplasmosis progression using liquid chromatography-mass spectrometry. Sci Rep 6: 19557

Publisher's note Springer Nature remains neutral with regard to jurisdictional claims in published maps and institutional affiliations. 